\title{
ON A CLASS OF SETS VIA GRILL : A DECOMPOSITION OF CONTINUITY
}

\author{
Dhananjoy Mandal and M. N. Mukherjee
}

\begin{abstract}
In the present article, a class of sets, called $\mathcal{G}$-semiclosed sets, which is a subclass of the class of semi-closed sets of Levine [7], is introduced and studied in a grill topological space $(X, \tau, \mathcal{G})$, where $\mathcal{G}$ is a grill on $X$. Two types of functions are then introduced which ultimately lead us to achieve a new decomposition of a continuous function.
\end{abstract}

\section{INTRODUCTION AND PRELIMINARIES}

As is noticed from the literature, there has been a growing trend among some topologists to introduce and study different allied or weaker forms of open sets, motivating the investigation of the corresponding types of continuous-like functions between topological spaces. This again has given rise to different decompositions of continuous function. A classical example towards decomposition of continuity is the paper of $\mathrm{N}$. Levine [6], whereas a very recent attempt by Hatir and Jafari [5] with the same motivation has culminated in the introduction and study of $\Phi$-open sets, where $\Phi$ is a suitable operator. This operator $\Phi: P(X) \rightarrow P(X)$, where $X$ is a topological space was first defined in [9] in terms of grill; the latter concept being defined by Choquet [4] several decades back. Interestingly, it is found from subsequent investigations that the notion of grills as an appliance like nets and filters, turns out to be extremely useful towards the study of certain specific topological problems (see for instance [2], [3] and [12]). The definition of grill on a topological space, as

Key Words: Grill, semi-open set, $\mathcal{G}$-semiopen set, $\mathcal{G}$-semicontinuity, $\mathcal{G S}$-set, GS-continuity. 2010 Mathematics Subject Classification: 54A10, 54C08

Received: March, 2011.

Accepted: February, 2012. 
given by Choquet [4], goes as follows: A non-null collection $\mathcal{G}$ of subsets of a topological spaces $X$ is said to be a grill on $X$ if

(i) $\phi \notin \mathcal{G}$, (ii) $A \in \mathcal{G}$ and $A \subseteq B \subseteq X \Rightarrow B \in \mathcal{G}$, and (iii) $A, B \subseteq X$ and $A \cup B \in \mathcal{G} \Rightarrow A \in \mathcal{G}$ or $B \in \mathcal{G}$.

For a grill $\mathcal{G}$ on a topological space $(X, \tau)$, an operator $\Phi$ from the power set $P(X)$ of $X$ to $P(X)$ was defined in [9] in the following manner : For any $A \in P(X), \Phi(A)=\{x \in X: U \cap A \in \mathcal{G}$, for each open neighbourhood $U$ of $x\}$. Then the operator $\Psi: P(X) \rightarrow P(X)$, given by $\Psi(A)=A \bigcup \Phi(A)$ (for $A \subseteq X)$, was also shown in [9] to be a Kuratowski closure operator, defining a unique topology $\tau_{\mathcal{G}}$ ( say ) on $X$ such that $\tau \subseteq \tau_{\mathcal{G}}$.

As mentioned above, Hatir and Jafari [5] utilized the operator $\Phi$ to accomplish their decomposition of continuity. Our intention in the present article is to pursue the trend. To that end, we introduce to start with, a kind of closed set, termed $\mathcal{G}$-semiclosed, defined in terms of a grill $\mathcal{G}$ and the said operator $\Phi$. Among other things it is shown in Section 2 that every $\mathcal{G}$-semiclosed set is semi-closed [7], but is independent of the concept of $\mathcal{G}$-closedness ([5], [11]). We then take up the task of decomposition of continuity in Section 3. For that we have defined a type of sets, dubbed 9S-sets, and this has enabled us to achieve finally the desired decomposition. In the process we obtain a decomposition of openness of subsets in a grill topological space.

In what follows in this paper, a space $X$ will always be taken to stand for a topological space $(X, \tau)$. If $A \subseteq X$, we shall adhere to the usual convention to write $\operatorname{int}(A)$ and $\operatorname{cl}(A)$ respectively for interior and closure of a set $A$ in $(X, \tau)$. Whenever we say that a subset $A$ of a space $X$ is open (resp. closed), it will mean that $A$ is open (resp. closed) in $(X, \tau)$. For open and closed sets with

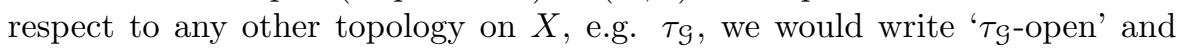
' $\tau_{\mathcal{G}}$-closed'. A subset $A$ of $(X, \tau)$ is said to be semi-open [7] if $A \subseteq \operatorname{cl}(\operatorname{int}(A))$. A topological space $(X, \tau)$ with a grill $\mathcal{G}$ on $X$, denoted by $(X, \bar{\tau}, \mathcal{G})$, will be called a grill topological space. We now recall a few results from [9] to be used in the sequel.

Theorem 1.1. Let $(X, \tau)$ be a topological space and $\mathcal{G}$ be grill on $X$. Then for any $A, B \subseteq X$ the following hold:

(a) $A \subseteq B \Rightarrow \Phi(A) \subseteq \Phi(B)$.

(b) $\Phi(A \cup B)=\Phi(A) \bigcup \Phi(B)$.

(c) $\Phi(\Phi(A)) \subseteq \Phi(A)=\operatorname{cl}((\Phi(A))) \subseteq \operatorname{cl}(\mathrm{A})$.

Theorem 1.2. Let $\mathcal{G}$ be grill on a topological space $(X, \tau)$. If $U \in \tau$, then 
$U \cap \Phi(A)=U \cap \Phi(U \cap A)$, for any $A \subseteq X$.

\section{SEMI-OPEN SETS WITH RESPECT TO A GRILL}

We begin by introducing a generalized class of semi-open sets in terms of grills as follows.

Definition 2.1. A subset $A$ of a grill topological space $(X, \tau, \mathcal{G})$ is said to be semi-open with respect to the grill $\mathcal{G}$ or simply $\mathcal{G}$-semiopen (resp. $\mathcal{G}$-open [11] or $\Phi$-open[5]) if $A \subseteq \Psi(\operatorname{int}(A))$ (resp. $A \subseteq \operatorname{int}(\Phi(A))$ ). The complement of a $\mathcal{G}$-semiopen (resp. $\mathcal{G}$-open or $\Phi$-open) set is called a $\mathcal{G}$-semiclosed (resp. G-closed or $\Phi$-closed) set.

\section{Remarks 2.2.}

(a) The Example 2.1 in [5] for showing the mutual independence of the concept of openness and $\Phi$-openness is wrong. Actually the collection $\mathcal{G}$ taken in the said example is not at all a grill. We give an example here (see Example 2.3) to establish the mutual independence of openness and $\Phi$-openness.

(b) Every $\mathcal{G}$-semiopen set is semi-open. In fact, let $A$ be $\mathcal{G}$-semiopen in $(X, \tau, \mathcal{G})$. Then $A \subseteq \Psi(\operatorname{int}(A))=\operatorname{int}(A) \cup \Phi(\operatorname{int} A)) \subseteq \operatorname{int}(A) \cup \operatorname{cl}(\operatorname{int}(A))$ (by Theorem 1.1 $)=\operatorname{cl}(\operatorname{int}(A))$. This shows that $A$ is semi-open. That the converse is false is shown below (see Example 2.4).

(c) The concepts of $\mathcal{G}$-semiopen and $\mathcal{G}$-open sets are independent of each other (see Example 2.5).

\section{Examples 2.3.}

(i) Let $X=\{a, b, c\}, \tau=\{\phi,\{a\},\{b, c\}, X\}$ and $\mathcal{G}=\{\{a\},\{a, c\},\{a, b\}, X\}$. Then $(X, \tau)$ is a topological space and $\mathcal{G}$ is a grill on $X$. Take $U=\{b, c\} \in \tau$. But $\Phi(U)=\phi$ so that $U$ is not $\Phi$-open.

(ii) Let $X=\{a, b, c\}, \tau=\{\phi,\{a\},\{b, c\}, X\}$ and $\mathcal{G}=\{\{a\},\{c\},\{a, c\},\{a, b\}$, $\{b, c\}, X\}$. Then $(X, \tau)$ is a topological space and $\mathcal{G}$ is a grill on $X$. Take $A=$ $\{a, c\}$. Then $\Phi(A)=X$, so that $A$ is $\Phi$-open but $A$ is not open in $(X, \tau)$.

Example 2.4. Let $X=\{a, b, c\}, \tau=\{\phi,\{b\},\{c\},\{b, c\}, X\}$ and $\mathcal{G}=\{\{a\}$, $\{a, b\},\{a, c\}, X\}$. Then $(X, \tau)$ is a topological space and $\mathcal{G}$ is a grill on $X$. Suppose $A=\{a, c\}$. Then $A$ is semi-open but it is not $\mathcal{G}$-semiopen. In fact, $\operatorname{int}(A)=\{c\}$ and $\Phi(\operatorname{int}(A))=\phi$, so that $\Psi(\operatorname{int}(A))=\{c\} \nsupseteq A$.

\section{Examples 2.5.}

(i) Consider the grill topological space $(X, \tau, \mathcal{G})$ where $X=\{a, b, c\}, \tau=$ $\{\phi,\{a\},\{b\},\{a, b\}, X\}$ and $\mathcal{G}=\{\{c\},\{a, c\},\{b, c\}, X\}$. Suppose $A=\{a, b\}$. 
Then $\Phi(A)=\phi$ so that $A$ is not $\Phi$-open. But $A$ is $\mathcal{G}$-semiopen as $\operatorname{int}(A)=$ $\{\mathrm{a}, \mathrm{b}\}$ and $\Phi(\operatorname{int}(\{a, b\}))=\phi$, so that $\Psi(\operatorname{int}(\{a, b\}))=\{a, b\}=A$.

(ii) Let $X=\{a, b, c\}, \tau=\{\phi,\{a\},\{b, c\}, X\}$ and consider the grill $\mathcal{G}=\{\{a\},\{c\}$, $\{a, b\},\{a, c\},\{b, c\}, X\}$ on $X$. Suppose $A=\{a, c\}$. Then $A$ is $\mathcal{G}$-open but not $\mathcal{G}$-semiopen. In fact, $\Phi(A)=\{a, b, c\}=X \Rightarrow A \subseteq \operatorname{int}(\Phi(A)) \Rightarrow A$ is $\mathcal{G}$-open; again, $\operatorname{int}(A)=\{a\}, \Phi(\{a\})=\{a\}$ and $\Psi(\operatorname{int}(\{a\}))=\{a\} \nsupseteq A \Rightarrow A$ is not $\mathcal{G}$-semiopen.

Definition 2.6. [10] Let $X$ be a nonempty set and $(\phi \neq) A \subseteq X$. Then

$$
[A]=\{B \subseteq X: A \bigcap B \neq \phi\}
$$

is a grill on $X$, called the principal grill generated by $A$.

Remark 2.7. In the case of the principal grill $[X]$ generated by $X$, it is known $[10]$ that $\tau=\tau_{[X]}$, so that any $[X]$-semiopen set becomes simply a semi-open set and vice-versa.

Theorem 2.8. Let $(X, \tau)$ be a topological space and $\mathcal{G}$ be a grill on $X$. Then a subset $A$ of $X$ is $\mathcal{G}$-semiopen iff $\Psi(A)=\Psi(\operatorname{int}(A))$.

Proof. Let $A$ be $\mathcal{G}$-semiopen. Then as $\Psi$ is monotonic and idempotent, $\Psi(A) \subseteq \Psi(\Psi(\operatorname{int}(A)))=\Psi(\operatorname{int}(A)) \subseteq \Psi(A) \Rightarrow \Psi(A)=\Psi(\operatorname{int}(A))$.

The converse is trivial.

Theorem 2.9. Let $(X, \tau)$ be a topological space and $\mathcal{G}$ be a grill on $X$. If a subset $A$ of $X$ is $\mathcal{G}$-semiclosed, then $\operatorname{int}(\Psi(A)) \subseteq A$.

Proof. Suppose $A$ is $\mathcal{G}$-semiclosed. Then $X \backslash A$ is $\mathcal{G}$-semiopen and hence $X \backslash A \subseteq \Psi(\operatorname{int}(X \backslash A)) \subseteq \operatorname{cl}(\operatorname{int}(X \backslash A))=X \backslash \operatorname{int}(\operatorname{cl}(A)) \subseteq X \backslash \operatorname{int}(\Psi(A)) \Rightarrow$ $\operatorname{int}(\Psi(A)) \subseteq A$.

Remark 2.10. The converse of the above theorem is false as shown in the following example.

Example 2.11. Consider the grill topological space $(X, \tau, \mathcal{G})$ where $X=$ $\{a, b, c\}, \tau=\{\phi,\{a\},\{b, c\}, X\}$ and $\mathcal{G}=\{\{a\},\{a, b\},\{a, c\}, X\}$. Suppose $A=$ $\{a, c\}$. Then $A$ is not $\mathcal{G}$-semiclosed but $\operatorname{int}(\Psi(A)) \subseteq A$. In fact, $\Phi(A)=\{a\}$. Thus $\Psi(A)=A \cup \Phi(A)=\{a, c\}$ and hence $\operatorname{int}(\Psi(A)) \subseteq A$. Again $X \backslash A=$ $\{b\}$. Thus $\operatorname{int}(\{b\})=\phi$ so that $\Psi(\operatorname{int}\{b\})=\phi \nsupseteq\{b\}=X \backslash A$ and hence $X \backslash A$ is not $\mathcal{G}$-semiopen $\Rightarrow A$ is not $\mathcal{G}$-semiclosed. 
Theorem 2.12. Let $(X, \tau, \mathcal{G})$ be a grill topological space and $A$ be a subset of $X$ such that $X \backslash \operatorname{int}(\Psi(A))=\Psi(\operatorname{int}(X \backslash A))$. Then $A$ is $\mathcal{G}$-semiclosed iff $\operatorname{int}(\Psi(A)) \subseteq A$.

Proof. One part follows from Theorem 2.9 .

Conversely, suppose that $\operatorname{int}(\Psi(A)) \subseteq A$. Then $X \backslash A \subseteq X \backslash \operatorname{int}(\Psi(A))=$ $\Psi(\operatorname{int}(X \backslash A)) \Rightarrow X \backslash A$ is $\mathcal{G}$-semiopen $\Rightarrow A$ is $\mathcal{G}$-semiclosed.

Theorem 2.13. Let $(X, \tau)$ be a space and $\mathcal{G}$ be a grill on $X$. Then a subset $A$ of $X$ is $\mathcal{G}$-semiopen iff there exists a $U \in \tau$ such that $U \subseteq A \subseteq \Psi(U)$.

Proof. Suppose $A$ is $\mathcal{G}$-semiopen. Then $A \subseteq \Psi(\operatorname{int}(A))$. Put $\operatorname{int}(A)=U$. Then $U \subseteq A \subseteq \Psi(U)$, where $U \in \tau$.

Conversely, let $U \subseteq A \subseteq \Psi(U)$ for some $U \in \tau$. Now $U \subseteq A \Rightarrow U \subseteq \operatorname{int}(A) \Rightarrow$ $\Psi(U) \subseteq \Psi(\operatorname{int}(A)) \Rightarrow A \subseteq \Psi(\operatorname{int}(A)) \Rightarrow A$ is $\mathcal{G}$-semiopen.

Remark 2.14. At this point it is quite pertinent to raise the question whether the semi-openness of a set in $\left(X, \tau_{\mathcal{G}}\right)$ is same as the $\mathcal{G}$-semiopenness of the set in $(X, \tau, \mathcal{G})$. The following example answers the question in the negative.

Example 2.15. Consider the grill topological space $(X, \tau, \mathcal{G})$, where $X=$ $\{a, b, c\}, \tau=\{\phi,\{a\},\{b, c\}, X\}$ and $\mathcal{G}=\{\{a\},\{c\},\{a, c\},\{a, b\},\{b, c\}, \mathrm{X}\}$. Then $\tau_{\mathcal{G}}=\{\phi,\{a\},\{c\},\{a, c\},\{b, c\}, X\}$. Here $A=\{a, c\}$ is open and hence semi-open in $\left(X, \tau_{\mathcal{G}}\right)$. But $A$ is not $\mathcal{G}$-semiopen in $(X, \tau, \mathcal{G})$.

Theorem 2.16. Let $(X, \tau)$ be a space and $\mathcal{G}$ be a grill on $X$. Let $A, B$ be subsets of $X$ such that $A \subseteq B \subseteq \Psi(A)$. If $A$ is $\mathcal{G}$-semiopen, then $B$ is also $\mathcal{G}$-semiopen.

Proof. Let $A$ be $\mathcal{G}$-semiopen. Then there exists $U \in \tau$ such that $U \subseteq A \subseteq$ $\Psi(U) \Rightarrow U \subseteq A \subseteq B \subseteq \Psi(A) \subseteq \Psi(\Psi(U))=\Psi(U)$ and hence $U \subseteq B \subseteq \Psi(U)$ for some $U \in \tau \Rightarrow B$ is $\mathcal{G}$-semiopen.

Theorem 2.17. Let $(X, \tau)$ be a space and $\mathcal{G}$ be a grill on $X$.

(a) If $\left\{U_{\alpha}: \alpha \in \Lambda\right\}$ is a family of $\mathcal{G}$-semiopen sets, then $\bigcup\left\{U_{\alpha}: \alpha \in \Lambda\right\}$ is G-semiopen.

(b) If $A(\subseteq X)$ is $\mathcal{G}$-semiopen and $U \in \tau$, then $A \cap U$ is $\mathcal{G}$-semiopen.

Proof. (a) Suppose $U_{\alpha}$ is G-semiopen, for each $\alpha \in \Lambda$. Then $U_{\alpha} \subseteq \Psi\left(\operatorname{int}\left(U_{\alpha}\right)\right)$, for each $\alpha \in \Lambda \Rightarrow \bigcup_{\alpha \in \Lambda} U_{\alpha} \subseteq \bigcup_{\alpha \in \Lambda} \Psi\left(\operatorname{int}\left(U_{\alpha}\right)\right) \subseteq \Psi\left(\operatorname{int} \bigcup_{\alpha \in \Lambda}\left(U_{\alpha}\right)\right)$. This shows that $\bigcup\left\{U_{\alpha}: \alpha \in \Lambda\right\}$ is $\mathcal{G}$-semiopen.

(b) Let $A$ be $\mathcal{G}$-semiopen and $U \in \tau$. Then $A \subseteq \Psi(\operatorname{int}(A))$. Now, $A \cap U \subseteq$ $\Psi(\operatorname{int}(A)) \bigcap U=(\operatorname{int}(A) \bigcup \Phi(\operatorname{int}(A))) \cap U=(\operatorname{int}(A) \cap U) \bigcup(\Phi(\operatorname{int}(A)) \bigcap U) \subseteq$ $\operatorname{int}(A \cap U) \bigcup \Phi(\operatorname{int}(A) \cap U)$ (using Theorem 1.2) $=\operatorname{int}(A \cap U) \bigcup \Phi(\operatorname{int}(A \cap U))$ 
$=\Psi(\operatorname{int}(A \cap U)) \Rightarrow A \bigcap U$ is $\mathcal{G}$-semiopen.

\section{G-SEMICONTINUOUS AND G-SEMICLOSED FUNCTIONS}

Definition 3.1. A function $f:(X, \tau, \mathcal{G}) \rightarrow(Y, \sigma)$ is said to be $\mathcal{G}$-semicontinuous (resp. semi-continuous [7]) if $f^{-1}(V)$ is $\mathcal{G}$-semiopen (resp. semi-open) for each $V \in \sigma$.

Remark 3.2. It is easy to see that

$$
\text { continuity } \Rightarrow \mathcal{G} \text {-semicontinuity } \Rightarrow \text { semi-continuity. }
$$

But the reverses of the above implications are false as is shown below.

\section{Examples 3.3.}

(i) Let $X=\{a, b, c, d\}, \tau=\{\phi,\{d\},\{a, c\},\{a, c, d\}, X\}$

and $\mathcal{G}=\{\{d\},\{a, d\},\{b, d\},\{c, d\},\{a, b, d\},\{a, c, d\},\{b, c, d\}, X\}$.

We define a function $f:(X, \tau, \mathcal{G}) \rightarrow(X, \tau)$ as follows:

$$
f(a)=f(b)=f(c)=d \text { and } f(d)=c .
$$

Then it is easy to see that $\mathrm{f}$ is semi-continuous but not $\mathcal{G}$-semicontinuous (in fact, $A=\{d\} \in \tau$ and $f^{-1}(\{d\})=\{a, b, c\}$ is not $\mathcal{G}$-semiopen).

(ii) Let $X=\{a, b, c, d\}, \tau=\{\phi,\{a\},\{b, d\},\{a, b, d\}, X\}$

and $\mathcal{G}=\{\{a\},\{a, b\},\{a, c\},\{a, d\},\{a, b, c\},\{a, b, d\},\{a, c, d\}, X\} ; Y=\{x, y\}$ and $\sigma=\{\phi,\{x\}, Y\}$. We define a function $f:(X, \tau, \mathcal{G}) \rightarrow(Y, \sigma)$ as

$$
f(a)=f(c)=x \text { and } f(b)=f(d)=y .
$$

Then $\mathrm{f}$ is not continuous but it is $\mathcal{G}$-semicontinuous. In fact, it is easy to check that $A \subseteq \Psi(\operatorname{int}(A))$, where $A=f^{-1}(\{x\})$.

Theorem 3.4. For a function $f:(X, \tau, \mathcal{G}) \rightarrow(Y, \sigma)$, the following are equivalent:

(a) $\mathrm{f}$ is $\mathrm{G}$-semicontinuous.

(b) The inverse image of each closed subset of $Y$ is $\mathcal{G}$-semiclosed.

(c) For each $x \in X$ and each $V \in \sigma$ containing $f(x)$, there exists a $\mathcal{G}$-semiopen set $U$ containing $x$ such that $f(U) \subseteq V$.

Proof. (a) $\Leftrightarrow(\mathbf{b})$ : It is clear.

(a) $\Rightarrow(\mathbf{c})$ : Let $V \in \sigma$ and $f(x) \in V(x \in X)$. Then by (a), $f^{-1}(V)$ is a $\mathcal{G}$ semiopen set containing $x$. Taking $f^{-1}(V)=U$, we have $x \in U$ and $f(U) \subseteq V$. (c) $\Rightarrow\left(\right.$ a): Let $V$ be any open set in $Y$ and $x \in f^{-1}(V)$. Then $f(x) \in V \in \sigma$ and hence by $(\mathrm{c})$, there exists a $\mathcal{G}$-semiopen set $U$ containing $x$ such that 
$f(U) \subseteq V$. Now $x \in U \subseteq \Psi(\operatorname{int}(U)) \subseteq \Psi$ (int $\left.\left(f^{-1}(V)\right)\right)$. This shows that $f^{-1}(V) \subseteq \Psi\left(\operatorname{int}\left(f^{-1}(V)\right)\right)$. Thus $f$ is $\mathcal{G}$-semicontinuous.

Theorem 3.5. A function $f:(X, \tau, \mathcal{G}) \rightarrow(Y, \sigma)$ is $\mathcal{G}$-semicontinuous iff the graph function $g: X \rightarrow X \times Y$, defined by $g(x)=(x, f(x))$, for each $x \in X$, is $\mathcal{G}$-semicontinuous.

Proof. Suppose that $f$ is $\mathcal{G}$-semicontinuous. Let $x \in X$ and $W$ be any open set in $X \times Y$ containing $g(x)$. Then there exist $U \in \tau$ and $V \in \sigma$ such that $g(x)=(x, f(x)) \in U \times V \subseteq W$. Since $f$ is $\mathcal{G}$-semicontinuous, there exists a $\mathcal{G}$-semiopen set $G$ of $X$ containing $x$ such that $f(G) \subseteq V$. By Theorem 2.17 (b), $G \cap U$ is $\mathcal{G}$-semiopen and $g(G \cap U) \subseteq U \times V \subseteq W$. This shows that $g$ is G-semicontinuous.

Conversely, suppose that $g$ is $\mathcal{G}$-semicontinuous. Let $x \in X$ and $V$ be any open set in $Y$ containing $f(x)$. Then $X \times V$ is open in $X \times Y$ and by $\mathcal{G}_{-}$ semicontinuity of $g$, there exists a $\mathcal{G}$-semiopen set $U$ containing $x$ such that $g(U) \subseteq X \times V$. Thus we have $f(U) \subseteq V$ and hence $f$ is $\mathcal{G}$-semicontinuous.

Definition 3.6. Let $(X, \tau)$ be a topological space and $(Y, \sigma, \mathcal{G})$ a grill topological space. A function $f:(X, \tau) \rightarrow(Y, \sigma, \mathcal{G})$ is said to be $\mathcal{G}$-semiopen (resp. $\mathcal{G}$-semiclosed) if for each $U \in \tau$ (resp. closed set $U$ in $(X, \tau)), f(U)$ is $\mathcal{G}$ semiopen (resp. $\mathcal{G}$-semiclosed) in $(Y, \sigma, \mathcal{G})$.

Definition 3.7.[1] A function $f:(X, \tau) \rightarrow(Y, \sigma)$ is said to be semi-open (resp. semi-closed) if for each $U \in \tau$ (resp. for each closed set $U$ in $(X, \tau)$ ), $f(U)$ is semi-open (resp. semi-closed) in $(Y, \sigma)$.

\section{Remarks 3.8.}

(a) Every open function is $\mathcal{G}$-semiopen, but the converse is false as is shown in Example 3.9 .

(b) Every $\mathcal{G}$-semiopen (resp. $\mathcal{G}$-semiclosed) function is semi-open (resp. semiclosed); that the converses are false is shown in Example 3.10.

Example 3.9. Let $X=\{a, b, c\}, \tau=\{\phi,\{a, b\}, X\}, \sigma=\{\phi,\{a\},\{c\},\{a, c\}, X\}$ and $\mathcal{G}=\{\{a\},\{a, b\},\{a, c\}, X\}$. Then the identity function $f:(X, \tau) \rightarrow$ $(X, \sigma, \mathcal{G})$ is $\mathcal{G}$-semiopen but it is not open.

\section{Examples 3.10.}

(i) Let $X=\{a, b, c\}, \tau=\{\phi,\{a, b\}, X\}$ and $\sigma=\{\phi,\{a\}, X\}, \mathcal{G}=\{\{b\},\{a, b\}$, $\{b, c\}, X\}$. Then the identity function $f:(X, \tau) \rightarrow(X, \sigma, \mathcal{G})$ is semi-open, but not $\mathcal{G}$-semiopen.

(ii) Let $X=\{a, b, c\}, \tau=\{\phi,\{a\}, X\}$ and $\sigma=\{\phi,\{b\},\{c\},\{b, c\}, X\}, \mathcal{G}=$ 
$\{\{a\},\{a, b\},\{a, c\}, X\}$. We define a function $f:(X, \tau) \rightarrow(X, \sigma, \mathcal{G})$ as follows:

$$
f(a)=a, f(b)=f(c)=b .
$$

Then $f$ is semi-closed, but it is not $\mathcal{G}$-semiclosed.

Theorem 3.11. A function $f:(X, \tau) \rightarrow(Y, \sigma, \mathcal{G})$ is $\mathcal{G}$-semiopen iff for each $x \in X$ and each neighbourhood $U$ of $x$, there exists a $\mathcal{G}$-semiopen set $V$ in $Y$ such that $f(x) \in V \subseteq f(U)$.

Proof. Suppose that $f$ is a $\mathcal{G}$-semiopen function and let $x \in X$. Also let $U$ be any neighbourhood of $x$. Then there exists $G \in \tau$ such that $x \in G \subseteq U$. Since $f$ is $\mathcal{G}$-semiopen, $f(G)=V$ ( say) is $\mathcal{G}$-semiopen and $f(x) \in V \subseteq f(U)$. Conversely, suppose that $U$ is any open set in $X$. Then for each $x \in U$, there exists a $\mathcal{G}$-semiopen set $V_{x}$ such that $f(x) \in V_{x} \subseteq f(U)$. Thus $f(U)=\bigcup\left\{V_{x}\right.$ : $x \in U\}$ and hence by Theorem $2.17(\mathrm{a}), f(U)$ is $\mathcal{G}$-semiopen. This shows that $f$ is $\mathcal{G}$-semiopen.

Theorem 3.12. Let $f:(X, \tau) \rightarrow(Y, \sigma, \mathcal{G})$ be a $\mathcal{G}$-semiopen function. If $V$ is any subset of $Y$ and $F$ is a closed subset of $X$ containing $f^{-1}(V)$, then there exists a $\mathcal{G}$-semiopen set $H$ in $(Y, \sigma, \mathcal{G})$ containing $V$ such that $f^{-1}(H) \subseteq F$.

Proof. Suppose that $f$ is $\mathcal{G}$-semiopen. Let $V$ be any subset of $Y$ and $F$ be a closed subset of $X$ containing $f^{-1}(V)$. Then $X \backslash F$ is open in $(X, \tau)$ and hence by $\mathcal{G}$-semiopenness of $f, f(X \backslash F)$ is $\mathcal{G}$-semiopen. Thus $H=Y \backslash f(X \backslash F)$ is $\mathcal{G}$-semiclosed and consequently $f^{-1}(V) \subseteq F$ implies that $V \subseteq H$. Further we obtain that $f^{-1}(H) \subseteq F$.

If we set $\mathcal{G}=[X]$ in the above theorem, we have the following result which incidentally proves Theorem 3.1 of [8] (refer to Remark 2.7).

Corollary 3.13. Suppose a function $f: X \rightarrow Y$ is semi-open. Then for any subset $V$ of $Y$ and for any closed set $F$ of $X$ containing $f^{-1}(V)$, there exists a semi-closed set $H$ of $Y$ containing $V$ such that $f^{-1}(H) \subseteq F$.

Theorem 3.14. For any bijection $f:(X, \tau) \rightarrow(Y, \sigma, \mathcal{G})$ the following are equivalent:

(a) $f^{-1}:(Y, \sigma, \mathcal{G}) \rightarrow(X, \tau)$ is $\mathcal{G}$-semicontinuous.

(b) $f$ is $\mathcal{G}$-semiopen.

(c) $f$ is $\mathcal{G}$-semiclosed.

Proof. Obvious.

To accomplish the desired decomposition of continuity, we now define a type of sets and a kind of functions in terms of such sets. 
Definition 3.15. A subset $A$ of a grill topological space $(X, \tau, \mathcal{G})$ is said to be a 9S-set if $A=U \cap V$, where $U \in \tau$ and $\Psi(\operatorname{int} V)=\operatorname{int} V$.

The above definition incidentally gives a decomposition of openness of sets in a grill topological space as follows.

Theorem 3.16. Let $(X, \tau, \mathcal{G})$ be a grill topological space. Then a subset $A$ of $X$ is open in $X$ iff it is $\mathcal{G}$-semiopen and a $\mathcal{G S}$-set in $(X, \tau, \mathcal{G})$.

Proof. Let $A$ be open in $X$. Then $A$ is $\mathcal{G}$-semiopen as $A \subseteq \Psi(\operatorname{int}(A))$. Also $A$ can be expressed as $A=A \cap X$, where $A$ is open in $X$ and $\Psi(\operatorname{int} X)=\operatorname{int} X$. Thus $A$ is a 9 S-set.

Conversely, let $A$ be $\mathcal{G}$-semiopen and a GS-set. Thus $A \subseteq \Psi($ int $(A))=$ $\Psi(\operatorname{int}(U \cap V))$ where $U \in \tau$ and $\Psi(\operatorname{int} V)=\operatorname{int} V$. Now $A \subseteq U \cap A \subseteq$ $U \bigcap \Psi(\operatorname{int}(U \cap V))=U \bigcap \Psi(U \bigcap \operatorname{int}(V)) \subseteq U \bigcap \Psi(U) \bigcap \Psi(\operatorname{int}(V))=U \bigcap \operatorname{int}(V)$ $=\operatorname{int}(A)$. Hence $A$ is open in $X$.

Definition 3.17. A function $f:(X, \tau, \mathcal{G}) \rightarrow(Y, \sigma)$ is said to be $\mathcal{G S}$-continuous if for each $V \in \sigma, f^{-1}(V)$ is a $\mathcal{G S}$-set in $(X, \tau, \mathcal{G})$.

Using Theorem 3.16, we obtain the following decomposition of continuity: Theorem 3.18. Let $(X, \tau, \mathcal{G})$ be a grill topological space. Then for a function $f:(X, \tau, \mathcal{G}) \rightarrow(Y, \sigma)$, the following are equivalent:

(a) $f$ is continuous.

(b) $f$ is $\mathcal{G}$-semicontinuous and $\mathcal{G S}$-continuous.

\section{References}

[1] N. Biswas; On some mappings in topological spaces, Bull. Calcutta Math. 61 (1969), 127-135.

[2] K. C. Chattopadhyay, O. Njastad and W. J. Thron; Merotopic spaces and extensions of closure spaces, Canad. J. Math., (No 4) (1983), 613-629.

[3] K. C. Chattopadhyay and W. J. Thron; Extensions of closure spaces, Canad. J. Math., (No 6) (1977), 1277-1286.

[4] G. Choquet; Sur les notions de filtre et grille, Comptes Rendus Acad. Sci. Paris, 224 (1947), 171-173.

[5] E. Hatir and S. Jafari; On some new classes of sets and a new decomposition of continuty via grills, J. Advs. Math. Studies., 3(1) (2010), 33-40. 
[6] N. Levine; A decomposition of continuity in topological spaces, Amer Math. Monthly, 68 (1961), 36-41.

[7] N. Levine; Semi-open sets and semi-continuity in topological spaces, Amer Math. Monthly, 70(1963), 44-46.

[8] T. Noiri and B. Ahmad; A note on semi-open function, Mathematics Seminar Notes, 10 (1982) 437-441.

[9] B. Roy and M. N. Mukherjee; On a typical topology induced by a grill, Soochow J. Math., 33(4)(2007), 771-786.

[10] B. Roy and M. N. Mukherjee; Concerning topologies induced by principal grills, An. Stiint. Univ. AL. I. Cuza Iasi. Mat.(N. S.), 55 (2) (2009), 285-294.

[11] B. Roy, M. N. Mukherjee and S. K. Ghosh ; On a subclass of preopen sets via grills, Stud. Si Cercet. Stiint. Ser. Mat. Univ. Bacau, 18 (2008), 255-266.

[12] W. J. Thron; Proximity structures and grills, Math. Ann., 206 (1973), 35-62.

Dhananjoy Mandal,

Department of Pure Mathematics,

University of Calcutta,

35, Ballygunge Circular Road,

Kolkata- 700019 ,

India

Email: dmandal.cu@gmail.com

M. N. Mukherjee,

Department of Pure Mathematics,

University of Calcutta,

35, Ballygunge Circular Road,

Kolkata- 700019

India

Email: mukherjeemn@yahoo.co.in 